\title{
COMPORTAMENTO DIÁRIO AO LONGO DO PERÍODO DE ALIMENTAÇÃO DO PRIMEIRO ESTÁGIO DO SISTEMA FRANCÊS DE WETLAND VERTICAL, EM TERMOS DE REMOÇÃO DE MATÉRIA ORGÂNICA E AMÔNIA
}

\author{
Camila Maria Trein $^{1 *}$, Jorge Alejandro García Zumalacarregui ${ }^{2}$, Marcos von Sperling 3 \\ ${ }^{1}$ Programa de Pós-graduação em Engenharia Sanitária e Ambiental, Departamento de Engenharia Sanitária e Ambiental, \\ Universidade Federal de Minas Gerais, CEP 31270-901, Belo Horizonte - MG, Brasil
}

${ }^{2}$ Programa de Pós-graduação em Engenharia Sanitária e Ambiental, Departamento de Engenharia Sanitária e Ambiental, Universidade Federal de Minas Gerais, CEP 31270-901, Belo Horizonte - MG, Brasil / Universidade de Cuenca, Cuenca, Equador

${ }^{3}$ Programa de Pós-graduação em Engenharia Sanitária e Ambiental, Universidade Federal de Minas Gerais, CEP 31270-901, Belo Horizonte - MG, Brasil

*e-mail: camilatrein@yahoo.com.br

\section{RESUMO}

O objetivo desse trabalho foi avaliar o comportamento do potencial de nitrificação e remoção de matéria orgânica no primeiro estágio de um wetland construído vertical - sistema francês (WCV-SF), operando com dois leitos em paralelo, cada um com 7 dias de alimentação e 7 dias de repouso, sob condições climáticas brasileiras. A estação de tratamento avaliada está localizada no Centro de Pesquisa e Treinamento em Saneamento (CePTS) da Universidade Federal de Minas Gerais e Companhia de Saneamento de Minas Gerais, UFMG/COPASA, na cidade de Belo Horizonte, Brasil. Complementando o monitoramento rotineiro praticado há anos neste sistema, efetuou-se um monitoramento intensivo da qualidade do afluente e do efluente do WCV-SF durante uma semana, por meio de análises físico-químicas, através de coletas simples diárias nos tempos de 5, 10, 15, 20, 30, 40 e 50 minutos depois do início de uma batelada. Durante o período de monitoramento foi possível observar a eficiência dessa etapa associada à decomposição da matéria orgânica, permanecendo satisfatória mesmo em períodos de sobrecarga. Com concentrações positivas de oxigênio no efluente, o desempenho do sistema ao longo dos 7 dias de alimentação apresentou eficiência média de remoção e 69\% para DQO. Em termos de nitrogênio, a eficiência de remoção de $\mathrm{N}^{-\mathrm{NH}_{4}}{ }^{+}$foi de $58 \%$ e a produção de $\mathrm{N}^{-\mathrm{NO}_{3}}{ }^{-}$diminuiu com o avanço dos dias de alimentação, reportando $20 \mathrm{mg} \mathrm{L}^{-1}$ no $2^{\circ}$ dia e $11 \mathrm{mg} \mathrm{L}^{-1}$ no último. De uma forma geral, apesar de ter apenas duas unidades em paralelo, o sistema mostrou boa capacidade de receber esgoto bruto por um período de tempo maior de alimentação, reforçando os resultados obtidos no monitoramento rotineiro.

Palavras-chave: Esgoto bruto. Esgoto sanitário. Estratégias operacionais. Tratamento de esgotos.

\section{Introdução}

O primeiro estágio do wetland construído vertical utilizado para o tratamento de esgoto bruto, conhecido como sistema francês (WCV-SF), começou a ser empregado em locais de clima quente e frio, principalmente pela boa eficiência na degradação da matéria orgânica carbonácea $[1,2]$.

$\mathrm{Na}$ França, dados de monitoramento do primeiro estágio indicaram eficiências na ordem de $79 \%$ e $86 \%$ para demanda química de oxigênio (DQO) e sólidos suspensos totais (SST), respectivamente, trabalhando com carregamentos de aproximadamente de $300 \mathrm{~g} \mathrm{DQO} \mathrm{m}^{2} \mathrm{~d}^{-1}$ e $150 \mathrm{~g} \mathrm{SS} \mathrm{m}^{2} \mathrm{~d}^{-1} \mathrm{em}$ uma área de $1,2 \mathrm{~m}^{2}$ pessoa $^{-1}$ [1]. Eficiências similares foram encontradas por Morvannou et al. [3], avaliando 51 unidades (primeiro estágio) em operação na França, com valores médios de $77 \%$ para DQO e $83 \%$ para SST.

Esse desempenho de tratamento é garantido pela forma de operação das três unidades pertencentes ao primeiro estágio. Alternância de ciclos de alimentação por 3,5 a 4 dias e repouso por 7 dias é considerada importante para o controle do crescimento bacteriano e necessário para manter as condições aeróbias no filtro, permitindo assim, a mineralização da matéria orgânica proveniente da retenção dos sólidos suspensos contidos no afluente bruto [1] que se acumulam na superfície do sistema, formando uma camada de cerca de 2,5 $\mathrm{cm} \mathrm{ano}^{-1}$ [4].

Em termos de nitrogênio, o sistema apresenta algumas limitações, sendo identificada nitrificação em torno de $50 \%$ para cargas variando entre 25 a 30 g nitrogênio total Kjedahl (NTK) m $^{2}$ 
$\mathrm{d}^{-1}$ [1]. Vários são os fatores que mostram-se sensíveis a esse processo, como as condições operacionais (alternância dos filtros, estratégias de alimentação), taxa de transferência de oxigênio para o interior do maciço filtrante, composição do esgoto, considerações de projeto (altura do filtro, características do material filtrante, cargas hidráulicas, orgânicas) ou parâmetros externos (manutenção, clima) [4 - 9].

Bollet et al. [5] e Molle et al. [6] relatam condições opostas de operação (número de bateladas por dia) para o melhor desempenho dos sistemas em termos de remoção de matéria orgânica e transformação do nitrogênio. Segundo os autores, para obter melhores resultados na formação de nitrato, a estratégia indicada é a aplicação de maiores volumes em baixa frequência. Esse procedimento melhora a drenagem do líquido, obtendo uma melhor oxigenação do filtro, refletindo em um maior potencial de nitrificação na unidade. Opostamente, menores volumes em intervalos curtos conduzem a um maior teor de água no meio, prejudicando a nitrificação, porém contribuindo para a remoção da matéria orgânica.

Como forma de obter resultados satisfatórios que refletem na durabilidade e confiabilidade ao longo do tempo de operação, Torrens et al. [7] reforçam a necessidade de controlar os períodos de alimentação e repouso, conforme proposto em projeto.

Visto que essas recomendações de operação (3,5-4 dias de alimentação / 7 dias de repouso) são para as condições climáticas de países de clima frio, o objetivo desse estudo foi avaliar o potencial de nitrificação e remoção de matéria orgânica no primeiro estágio em um wetland construído vertical - sistema francês operando com um tempo maior no ciclo de operação de uma unidade ( 7 dias de alimentação / 7 dias de repouso), sob condições climáticas brasileiras. Ademais, ao invés de se contar com os tradiocionais três leitos em paralelo, que compõem o primeiro estágio do sistema francês, a presente pesquisa utilizou apenas dois leitos, o que representa uma economia de área de 1/3.

\section{Metodologia}

A estação de tratamento de esgoto (ETE) avaliada está localizada na cidade de Belo Horizonte, Minas Gerais, no Centro de Pesquisa e Treinamento em Saneamento (CePTS) da Universidade Federal de Minas Gerais e está dentro das dependências da ETE Arrudas, pertencente à Companhia de Saneamento de Minas Gerais (COPASA) (1953'42" S, $43^{\circ} 52^{\prime} 42^{\prime \prime} \mathrm{W}$ ).

A ETE em escala de demonstração é composta por um WCV-SF, destinado a receber uma parcela de esgoto bruto preliminarmente tratado por peneira ( $6 \mathrm{~mm}$ de abertura), desviado da ETE Arrudas. Na Figura 1 apresenta-se o fluxograma do sistema, juntamente com os pontos em que foram realizadas as amostragens, conforme segue: afluente WCV-SF e efluente do WCV-SF - unidade III.

\subsection{Características construtivas e operacionais do wetland construído vertical - sistema francês}

O WCV-SF, construído em 2007, foi dimensionado para tratar águas residuárias geradas por uma população equivalente de 100 habitantes (vazão média de $13 \mathrm{~m}^{3} \mathrm{~d}^{-1}$ ). Concebido de acordo com as recomendações e especificações do CEMAGREF/IRSTEA, o primeiro estágio possui 3 leitos filtrantes, cada um com área superficial de 29,4 $\mathrm{m}^{2}$ (largura de 3,1 m e comprimento de 9,4 m), totalizando $88,2 \mathrm{~m}^{2}\left(0,9 \mathrm{~m}^{2}\right.$ pessoa $\left.{ }^{-1}\right)$.

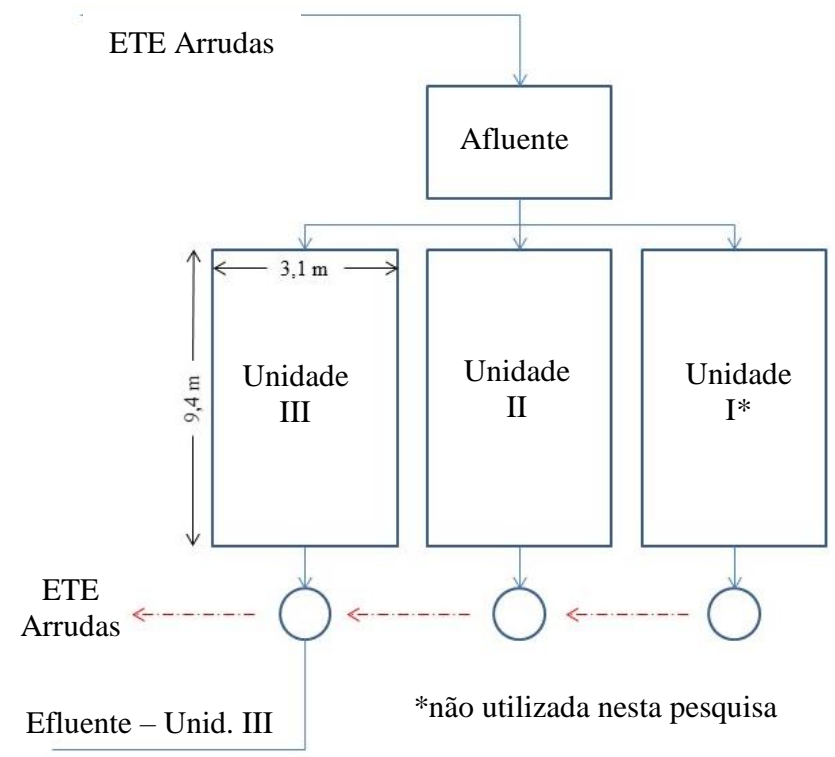

Figura 1 - Representação esquemática do primeiro estágio do wetland construído vertical - sistema francês.

Construída em alvenaria, as unidades foram preenchidas com $15 \mathrm{~cm}$ de brita 3 (19 a $50 \mathrm{~mm}$ ) na zona de drenagem (fundo), $15 \mathrm{~cm}$ de brita $1(4,8$ a $25 \mathrm{~mm})$ na camada intermediária e $40 \mathrm{~cm}$ de brita 0 (2,4 a 12,5 mm) na camada superior. Todas as unidades foram plantadas com capim - Tifton 85 (Cynodondactylon Pers.).

Além da alimentação intermitente de esgoto afluente sobre a superfície do wetland, que acontece a cada hora, ou seja, 24 pulsos $\mathrm{d}^{-1}$ de $0,53 \mathrm{~m}^{3}$, tem-se a alternância entre os filtros, que acontece a cada 7 dias. Atualmente apenas as unidades II e III estão em funcionamento (representando $0,6 \mathrm{~m}^{2}$ pessoa $^{-1}$ ), sendo que uma das unidades recebe esgoto durante uma semana enquanto a outra permanece em repouso nesse respectivo período.

\subsection{Monitoramento}

O sistema é objeto de monitoramento rotineiro desde seu início de funcionamento. Especificamente para este trabalho, foi realizado um monitoramento intensivo da qualidade do afluente e do efluente do WCV-SF durante uma semana no mês de março de 2017, por meio de análises físico-químicas, através de coletas 
simples realizadas no período da manhã nos dois pontos de amostragens, conforme destacado na Figura 1.

As amostras coletadas na saída do sistema tiveram como objetivo a caracterização do $1^{\circ}$ até o $7^{\circ}$ dia de alimentação, ou seja, durante 1 semana foram realizadas coletas de amostras nos tempos de $5,10,15,20,30,40$ e 50 minutos depois do início da alimentação (pulso) na unidade III. Por dia, foram coletadas 8 amostras, sendo 1 na entrada do sistema e 7 na saída, totalizando 56 amostras.

Os parâmetros analisados foram potencial hidrogeniônico $(\mathrm{pH})$, temperatura, oxigênio dissolvido (OD), DQO, NTK, nitrogênio amoniacal $\left(\mathrm{N}-\mathrm{NH}_{4}^{+}\right)$, nitrogênio nitrito $\left(\mathrm{N}-\mathrm{NO}_{2}{ }^{-}\right)$e nitrogênio nitrato $\left(\mathrm{N}-\mathrm{NO}_{3}{ }^{-}\right)$, seguindo recomendações do Standard Methods [10].

\section{Resultados e Discussões}

Os valores médios obtidos a partir da caracterização do afluente do wetland estão apresentados na Tabela 1.

Destaca-se que no $1^{\circ}$ dia de monitoramento as concentrações afluentes e efluentes do sistema para alguns parâmetros mostraram resultados fora do padrão de comportamento em relação ao monitoramento rotineiro, sendo assim descartados. Para os demais dias, com algumas variações, as características do esgoto permaneceram dentro da faixa típica de esgoto sanitário para as condições brasileiras [11].

Tabela 1. Valores médios da caracterização do afluente (esgoto bruto após tratamento preliminar) do sistema de tratamento avaliado - unidade III.

\begin{tabular}{|c|c|c|c|c|c|c|c|}
\hline \multirow{2}{*}{ Parâmetro } & \multicolumn{7}{|c|}{ Monitoramento (dias) } \\
\hline & $\mathbf{1}^{\mathrm{o}}$ & $2^{\circ}$ & $3^{\circ}$ & $4^{\circ}$ & $5^{\circ}$ & $6^{\circ}$ & $7^{\circ}$ \\
\hline $\mathrm{pH}$ & 6,78 & 7,01 & 7,24 & 7,3 & 7,33 & 7,44 & 7,30 \\
\hline $\mathrm{OD}\left(\mathrm{mg} \mathrm{L}^{-1}\right)$ & 0,76 & 0,50 & 0,22 & 0,86 & 1,13 & 0,15 & 0,17 \\
\hline Temperatura $\left({ }^{\circ} \mathrm{C}\right)$ & 27,1 & 27,2 & 27,8 & 27,8 & 24,7 & 26,4 & 25,4 \\
\hline DQO $\left(\mathrm{mg} \mathrm{L}^{-1}\right)$ & $*$ & 417 & 492 & 711 & 299 & 427 & 617 \\
\hline NTK $\left(\mathrm{mg} \mathrm{L}^{-1}\right)$ & 63,4 & 40,3 & 37,8 & 46,3 & 48,8 & 57,3 & 47,6 \\
\hline $\mathrm{N}-\mathrm{NH}_{4}{ }^{+}\left(\mathrm{mg} \mathrm{L}^{-1}\right)$ & $*$ & 33,5 & 27,4 & 36,6 & 40,5 & 34,1 & 26,5 \\
\hline $\mathrm{N}-\mathrm{NO}_{2}^{-}\left(\mathrm{mg} \mathrm{L}^{-1}\right)$ & 0,03 & 0,00 & 0,00 & 0,03 & 0,09 & 0,03 & 0,06 \\
\hline $\mathrm{N}-\mathrm{NO}_{3}^{-}\left(\mathrm{mg} \mathrm{L}^{-1}\right)$ & 0,77 & 0,00 & 0,00 & 0,16 & 1,42 & 0,00 & 0,54 \\
\hline
\end{tabular}

*valores não disponíveis.

Na Tabela 2 estão resumidos os resultados obtidos em termos de concentração efluente do primeiro estágio, e na Figura 2 é possível observar a eficiência dessa etapa associada à decomposição da matéria orgânica (DQO), que permaneceu satisfatória ao longo dos 7 dias de operação.

A vazão média afluente do wetland foi de $12,7 \mathrm{~m}^{3} \mathrm{~d}^{-1}$, o que resulta em uma taxa hidráulica aplicada na unidade III de 0,43 $\mathrm{m}^{3} \mathrm{~m}^{2} \mathrm{~d}^{-1}$. Baseado na concentração média de $494 \mathrm{mg}$ DQO L L ${ }^{-1}$ a unidade recebeu carregamento médio de $212 \mathrm{~g} \mathrm{DQO} \mathrm{m}^{2} \mathrm{~d}^{-1}$ durante o período de monitoramento. Apesar de estar acima das recomendações de $300 \mathrm{~g} \mathrm{DQO} \mathrm{m}^{2} \mathrm{~d}^{-1}$, no $4^{\circ}$ dia $\left(305 \mathrm{~g} \mathrm{DQO} \mathrm{m}^{2} \mathrm{~d}^{-}\right.$ ${ }^{1}$ ), a eficiência de remoção para DQO foi de $80 \%$ (Figura 2), mostrando que o sistema consegue receber sobrecargas pontuais sem afetar a taxa de remoção, conforme apontado por Morvannou et al. [3].

Porém, esses valores estão abaixo dos encontrados por Prost-Boucle e Molle [12] que, verificando o efeito de recirculação no primeiro estágio, observaram que, com carregamentos de até 450 g DQO $\mathrm{m}^{2} \mathrm{~d}^{-1}$, o desempenho na remoção do parâmetro permaneceu em $90 \%$, sendo que para $600 \mathrm{~g} \mathrm{DQO} \mathrm{m}^{2} \mathrm{~d}^{-1}$ as taxas de remoção foram em torno de $82 \%$ para as condições climáticas da França.

Tabela 2. Valores médios da caracterização do efluente do sistema de tratamento avaliado - unidade III.

\begin{tabular}{|c|c|c|c|c|c|c|c|}
\hline \multirow{2}{*}{ Parâmetros } & \multicolumn{7}{|c|}{ Monitoramento (dias) } \\
\hline & $\mathbf{1}^{\mathbf{o}}$ & $2^{\circ}$ & $3^{\circ}$ & $4^{\circ}$ & $5^{\circ}$ & $6^{\circ}$ & $7^{\circ}$ \\
\hline $\mathrm{pH}$ & 6,48 & 6,74 & 6,93 & 6,96 & 6,96 & 7,03 & 7,10 \\
\hline Temperatura $\left({ }^{\circ} \mathrm{C}\right)$ & 26,8 & 27,0 & 26,4 & 27,0 & 25,5 & 25,6 & 25,0 \\
\hline $\mathrm{OD}\left(\mathrm{mg} \mathrm{L}^{-1}\right)$ & 3,82 & 3,73 & 3,39 & 2,83 & 3,00 & 1,54 & 2,11 \\
\hline $\mathrm{DQO}\left(\mathrm{mg} \mathrm{L}^{-1}\right)$ & $*$ & 193 & 164 & 142 & 140 & 148 & 124 \\
\hline NTK ( mg L $\left.{ }^{-1}\right)$ & 10,6 & 11,6 & 17,6 & 15,3 & 21,0 & 18,8 & 20,5 \\
\hline $\mathrm{N}-\mathrm{NH}_{4}^{+}\left(\mathrm{mg} \mathrm{L}^{-1}\right)$ & 10,4 & 11,4 & 16,5 & 14,2 & 16,1 & 12,8 & 15,5 \\
\hline $\mathrm{N}-\mathrm{NO}_{2}^{-}\left(\mathrm{mg} \mathrm{L}^{-1}\right)$ & 0,25 & 0,61 & 0,20 & 0,79 & 0,15 & 3,12 & 0,08 \\
\hline $\mathrm{N}-\mathrm{NO}_{3}{ }^{-}\left(\mathrm{mg} \mathrm{L}^{-1}\right)$ & $*$ & 19,6 & 17,2 & 11,8 & 9,3 & 6,0 & 11,5 \\
\hline
\end{tabular}

*valores não disponíveis

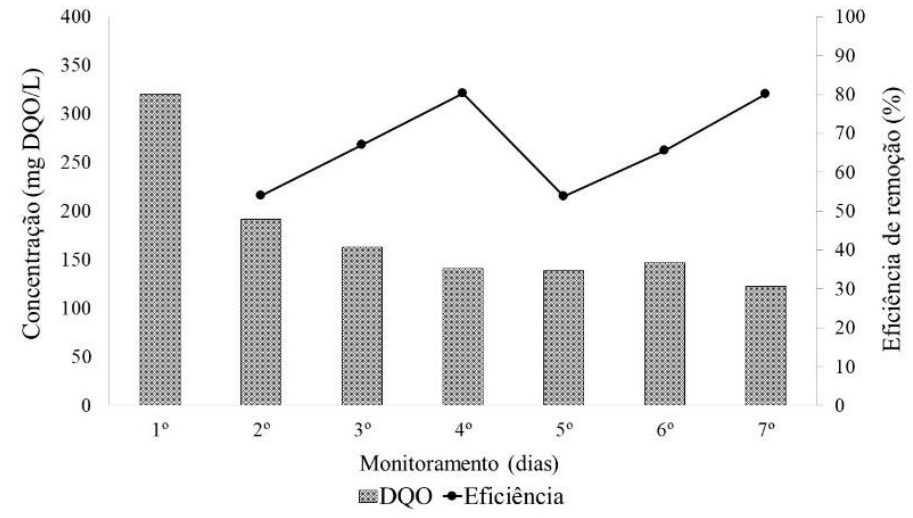

Figura 2 -Concentração média efluente e eficiência de remoção de DQO referente ao monitoramento de 7 dias.

De acordo com Molle et al. [1] e Morvannou et al. [3], o aumento do carregamento orgânico diminui o desempenho do tratamento em termos de NTK, refletindo nos limites que o sistema 
pode atingir em termos de renovação de oxigênio e adsorção de amônia.

Com valores médios de DQO efluente de $124 \mathrm{mg} \mathrm{L}^{-1}$ no $7^{\circ}$ dia de operação (Tabela 2 ) e remoção de $80 \%$, considera-se que o wetland apresentou desempenho satisfatório em termos de remoção de matéria orgânica, similar ao estudo realizado por Morvannou et al. [3], que reportam valores de $126 \mathrm{mg} \mathrm{L}^{-1} \mathrm{e}$ remoção de $77 \%$ para o primeiro estágio do sistema francês.

Como esperado, após a passagem do efluente pelo sistema, as concentrações de oxigênio dissolvido aumentaram (Figura 3, com medições ao longo da duração da batelada). Durante todo o período de operação da unidade III, as concentrações de oxigênio na saída foram positivas, representando até o $5^{\circ}$ dia as maiores concentrações (média 3,35 $\mathrm{mg} \mathrm{O}_{2} \mathrm{~L}^{-1}$ ), vindo a diminuir a partir do $6^{\circ}$ dia após o início da alimentação da unidade (média 1,82 $\mathrm{mg} \mathrm{O}_{2} \mathrm{~L}^{-1}$ ).

Diante de tais resultados, pode-se inferir que as condições estabelecidas favoreceram a ocorrência de processos aeróbios para a degradação da matéria orgânica e nitrificação. Contudo, a duração do regime operacional de 7 dias de alimentação sobrecarregou o sistema, que no $6^{\circ}$ dia apresentou dificuldades de drenagem do líquido que se acumulou na superfície, impedindo assim, o potencial de difusão de oxigênio no leito filtrante, justificando os menores valores nos últimos dias.

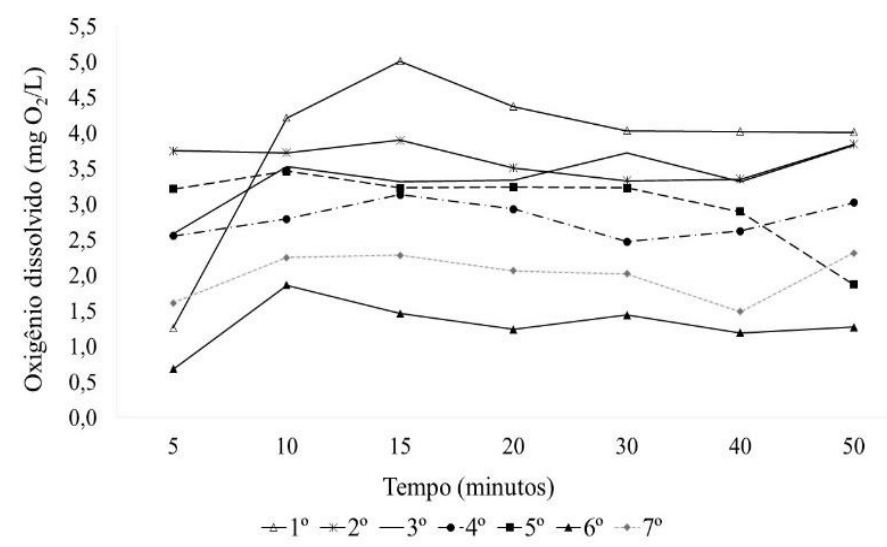

Figura 3 -Comportamento da concentração de $\mathrm{O}_{2}$ no efluente da unidade III durante 7 dias de monitoramento.

Em um estudo realizado na mesma planta de tratamento, após 2 anos do início de sua operação, foram identificadas concentrações médias de $5,1 \mathrm{mg} \mathrm{O}_{2} \mathrm{~L}^{-1}$ no efluente para o regime de operação de 24 pulsos $\mathrm{d}^{-1}$ consecutivamente por 2,5 dias de alimentação seguido de 4,5 dias de descanso [13]. É notório que com o passar do tempo (7 anos de operação) as condições de transferência de oxigênio para o leito filtrante foram afetadas, influenciadas principalmente pelo acúmulo de sólidos na parte superior do filtro (média de $6,6 \mathrm{~cm}$ ) e pela alimentação de uma mesma unidade feita por um período de tempo maior.

Torrens et al. [7] destacam que a falta do cumprimento com as recomendações de alimentação e períodos de repouso (3-4 dias de alimentação / 7 dias de repouso) para as condições climáticas da França aumentam o acúmulo de uma camada de lodo na superfície, trazendo problemas de entupimento na camada superior, fato não observado quando os critérios recomendados de operação são respeitados.

Além desse critério, Molle et al. [4] reforçam que as cargas hidráulicas e orgânicas, devem ser bem controladas a fim de favorecer a mineralização dessa camada. Caso contrário, temse limitações dos processos de transferência de oxigênio (convecção e difusão) pelo excesso de água na superfície, influenciando na performance do sistema.

A média da altura de lodo acumulado (de forma não homogênea) desde o início da operação da unidade III é menor que a reportada pelos sistemas operados na França $\left(2,5 \mathrm{~cm} \mathrm{ano}^{-1}\right)$, observação também relatada por Molle et al. [2] em duas unidades do primeiro estágio operados sob condições climáticas tropicais. Além das condições climáticas, que favorecem a mineralização dessa camada de lodo no Brasil, o esgoto afluente apresenta menor concentração de matéria orgânica (mais diluído), comparado com os gerados pela população das pequenas comunidades dos países europeus investigados.

A remoção de $\mathrm{NT}$ e $\mathrm{N}-\mathrm{NH}_{4}{ }^{+}$, bem como a produção de nitrogênio oxidado $\left(\mathrm{NO}_{\mathrm{x}}=\mathrm{N}-\mathrm{NO}_{3}{ }^{-}+\mathrm{N}-\mathrm{NO}_{2}{ }^{-}\right)$variou ao longo dos 7 dias de monitoramento (Figura 4). Para esse estudo, verificou-se que durante o período de monitoramento, dos $58 \%$ de $\mathrm{N}-\mathrm{NH}_{4}{ }^{+}$ removidos no sistema, uma parcela foi nitrificada (66\%), sendo

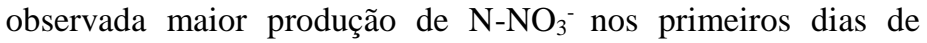
alimentação.

De acordo com Kantawanichkula, Kladpraserta e Brix [14], o baixo potencial para a nitrificação completa em wetlands verticais está associada à operação e à estrutura física dos sistemas, que não proporcionam tempo de detenção suficiente para permitir o contato com as bactérias que são responsáveis por promover a nitrificação. A rápida velocidade de fluxo limita a adsorção de amônia sobre o substrato que é nitrificada principalmente entre as bateladas e durante o período de repouso [6]. De acordo com Manjate et al. [15], os picos de vazão em um sistema não saturado são observados já nos primeiros 5 minutos, ou seja, depois de aplicado o esgoto na unidade o mesmo começa a sair após esse período.

No $4^{\circ}, 5^{\circ}$ e $6^{\circ}$ dia, a remoção de NT aumentou em comparação com os dias anteriores, enquanto que para $\mathrm{N}-\mathrm{NH}_{4}{ }^{+}$, a remoção se manteve estável com 61\%, 60\% e 62\%, respectivamente. Além disso, as concentrações de $\mathrm{NO}_{\mathrm{x}}$ produzidas diminuíram $\left(12,4,8,02\right.$ e 8,65 $\mathrm{mg} \mathrm{L}^{-1}$, respectivamente) em comparação com os dias anteriores. Esses resultados podem estar associados à desnitrificação. Muitos estudos relatam a baixa 
potencialidade de desnitrificação dos wetlands verticais [16 - 17], devido à predominância de condições aeróbias nos poros, após a passagem do líquido. Contudo, recentemente, utilizando técnicas de biologia molecular em nível de RNA em outro WCV, foram identificadas bactérias desnitrificantes ativas em todo o perfil vertical do sistema, sobretudo, quando o mesmo foi operado com alta carga orgânica a desnitrificação pode ocorrer desde a primeira camada do sistema [18].

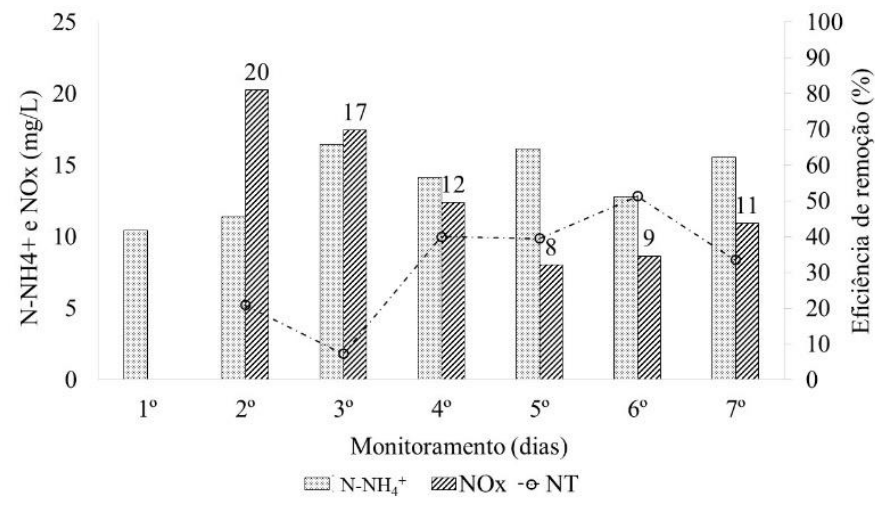

Figura 4 -Comportamento da série nitrogenada no efluente da unidade III durante 7 dias de monitoramento.

Como forma de aumentar o potencial de remoção do nitrogênio em wetland vertical, Silveira et al. [19] apresentam como estratégia a utilização da camada superior livre (condições aeróbias) e uma zona parcialmente saturada na parte inferior, visando criar condições aeróbicas/anóxicas em uma única unidade. Nos estudos destes autores foram obtidas eficiências da ordem de $56 \%$ para a nitrificação e $85 \%$ para a desnitrificação, com $25 \mathrm{~cm}$ de altura de leito saturado, para uma carga afluente de 14 g NTK $\mathrm{m}^{2} \mathrm{~d}^{-1}$.

Comparando com os resultados de Lana et al. [13] que pesquisou o mesmo sistema da presente pesquisa, o desempenho foi similar com o passar do tempo em termos de $\mathrm{N}_{-\mathrm{NH}_{4}}{ }^{+}(60 \%$ e $58 \%$ para este estudo) ao contrário dos resultados obtidos para DQO, que apresentou decaimento na eficiência de remoção de $80 \%$ para $69 \%$.

De forma a se ter um indicativo do potencial comportamento do sistema caso se adotasse a estratégia operacional dos franceses (3,5-4 dias de alimentação / 7 dias de repouso), os valores encontrados nesse estudo para o $4^{\circ}$ e $7^{\circ}$ dia de alimentação na mesma unidade apresentaram o seguinte comportamento em termos de porcentagem de remoção: 69\% para DQO nas duas condições e $60 \%$ e $58 \%$ para $\mathrm{N}_{-} \mathrm{NH}_{4}{ }^{+}$, respectivamente, com maior produção de $\mathrm{N}_{-} \mathrm{NO}_{3}{ }^{-}$nos três primeiros dias e maior remoção de NT a partir do $4^{\circ}$ dia.

De uma forma geral, mesmo com dificuldade de drenagem do líquido que se acumulou na superfície a partir do $6^{\circ}$ dia, o sistema mostrou boa capacidade para receber esgoto bruto por um período de alimentação maior. Porém, deve-se destacar que os valores obtidos no $4^{\circ}$ dia (com um período de alimentação de 7 dias) não necessariamente serão os mesmos que os obtidos com um efetivo período de alimentação de 4 dias, tal como adotado pelos franceses.

\section{Conclusões}

Considerando uma área superficial de $0,6 \mathrm{~m}^{2}$ pessoa $^{-1}(2$ leitos filtrantes) para o $1^{\circ}$ estágio do wetland construído vertical sistema francês tratando esgoto bruto em 24 bateladas por dia para condições operacionais de 7 dias de alimentação e 7 dias de repouso, sob condições climáticas brasileiras tem-se:

- Boa eficiência associada à decomposição da matéria orgânica, que permaneceu durante todo o período de monitoramento, mesmo em condições de sobrecarga, com remoção média de 69\% DQO;

- Dos $58 \%$ de $\mathrm{N}_{-} \mathrm{NH}_{4}{ }^{+}$removida no sistema, $66 \%$ foi nitrificada,

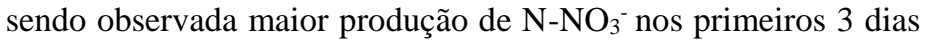
de operação;

- Mesmo com dificuldade de drenagem do líquido que se acumulou na superfície a partir do $6^{\circ}$ dia, o sistema mostrou boa capacidade para receber esgoto bruto por um período de alimentação de 7 dias consecutivos;

- Há a necessidade de um maior tempo de monitoramento para a caracterização da estratégia operacional referente ao período de alimentação e repouso.

\section{Agradecimentos}

Os autores agradecem as agências brasileiras CAPES, CNPq, FAPEMIG e FUNASA. Esta pesquisa faz parte de um programa internacional financiado pela Fundação Bill \& Melinda Gates para o projeto "Stimulating local innovation on sanitation for the urban poor in Sub-Saharan Africa and South-East Asia", sob a coordenação da Unesco-IHE Instituto de Educação da Água, Delft, Holanda.

\section{DAILY BEHAVIOR DURING THE FEEDING PERIOD OF THE FIRST STAGE OF A VERTICAL FLOW CONSTRUCTED WETLANDS - FRENCH SYSTEM IN TERMS OF ORGANIC MATTER AND AMMONIA REMOVAL}

ABSTRACT: The objective of this investigation was to evaluate the behaviour of the nitrification potential and organic matter removal in the first stage of a vertical flow constructed wetland French system (VCW-FS), operating with only two parallel beds, each with 7 days of feeding and 7 days of resting, under Brazilian climatic conditions. The treatment plant under evaluation is located at the Center for Research and Training in Sanitation (CePTS) of the Federal University of Minas Gerais (UFMG) and 
the Water and Sanitation Company of Minas Gerais (COPASA) in the city of Belo Horizonte, Brazil. In addition to the routine monitoring program implemented in this system over the past years, an intensive monitoring programme of the VCW-FS influent and effluent quality was carried out for one week, analysing physical-chemical parameters, through daily sample collections every $5,10,15,20,30,40$ and 50 minutes after the beginning of the batch loading. During the monitoring period, it was possible to observe the efficiency of this step associated with the organic matter decomposition and satisfactory results even under over loading conditions. With positive concentrations of oxygen in the effluent, the performance of the system during the 7 days of feeding presented an average removal efficiency of $69 \%$ for COD. In terms of nitrogen, $\mathrm{NH}_{4}{ }^{+}-\mathrm{N}$ removal efficiency was $58 \%$ and $\mathrm{NO}_{3}^{-}-\mathrm{N}$ production decreased with the feed days advance, reporting $20 \mathrm{mg} \mathrm{L}^{-1}$ on day 2 and $11 \mathrm{mg} \mathrm{L}^{-1}$ on the latter. In general, even using only two units in parallel, the system showed good capacity to receive raw for a longer period of feeding, reinforcing the results obtained in the routine monitoring.

Keywords: Raw sewage. Operational strategies. Sewage treatment.

\section{Referências}

[1] MOLLE, P.; LIÉNARD, A.; BOUTIN, C.; MERLIN, G.; IWEMA, A. How to treat raw sewage with constructed wetlands: an overview of the French systems. Water Science \& Technology, vol. 51, nº 9, p. 11-21, 2005.

[2] MOLLE, P., LOMBARD LATUNE, R., RIEGEL, C., LACOMBE, G., ESSER, D. French vertical-flow constructed wetland design: adaptations for tropical climates. Water Science \& Technology, p. 1516-1523, 2015.

[3] MORVANNOU, A.; FORQUET, N.; MICHEL, S.; TROESCH, S.; MOLLE, $P$. Treatment performances of French constructed wetlands: results from a data base collected over the last 30 years. Water Science \& Technology, vol. 71.9, 2015

[4] MOLLE, P. French vertical flow constructed wetlands: a need of a better understanding of the role of the deposit layer. Water Science \& Technology, vol. 69.1, p. 106-112, 2014.

[5] BOllet, M., SCHWAGER, A., EUGSTER, J., MOTTIER, V. Dynamic behavior of intermittent buried filters. Water Science \& Technology, vol. 28, $\mathrm{n}^{\circ} 10$, p. $99-107,1993$

[6] MOLLE, P.; LIÉNARD, A.; GRASMICK, A.; IWEMA, A. Effect of reed sand feeding operations on hydraulic behavior of vertical flow constructed wetlands under hydraulic overloads. Water Research, vol. 40, n³ 3, p. 606-612, 2006.

[7] TORRENS, A.; MOLLE, P.; BOUTIN, C.; SALGOT, M. Impact of design and operation variables on the performance of vertical-flow constructed wetland sand intermittent sand filters treating pond effluent. Water Research, vol. 43, p. 18511858,2009

[8] MILLOT, Y.; TROESCH, S.; ESSER, D.; MOLLE, P.; MORVANNOU, A.; GOURDON, R.; ROUSSEAU, D. Effects of design and operation al parameters on ammonium removal by single-stage French vertical flow filters treating raw domestic wastewater. Ecological Engineering, vol. 97, p. 516-523, 2016.
[9] NAKAMURA, K.; HATAKEYAMA, R.; TANAKA, N.; TAKISAWA, K., TADA, C.; NAKANO, K. A novel design for a compact constructed wetland introducing multi-filtration layers coupled with subsurface superficial space. Ecological Engineering, vol. 100, p. 99-106, 2016.

[10] APHA/AWWA/WEF. Standard methods for the examination of water and wastewater. $21^{\circ}$.ed. Washington, DC, 2011.

[11] VON SPERLING, M. Introdução à qualidade das águas e ao tratamento de esgotos. $4^{\text {a }}$ ed., Belo Horizonte: Editora UFMG, 472 p., 2014.

[12] PROST-BOUCLE, S E MOLLE, P. Recirculation on a single stage of vertical flow constructed wetland: Treatment limit sand operation modes. Ecological Engineering, vol. 43, p. 81-84, 2012

[13] LANA, L. C. O.; MORAES, D. C.; VON SPERLING, M.; MORATO, M. L.; VASCONCELLOS, G. R.; PARAENSE, M. O.; MOREIRA, T. P. A. Performance of single stage vertical flow constructed wetland system treating raw domestic sewage in Brazil. Water Science \& Technology, vol. 68, p. 1599. 2013.

[14] KANTAWANICHKUL, S.; KLADPRASERT, S., BRIX, H. Treatment of high-strength wastewater in tropical vertical flow constructed wetlands planted with Typha angustifolia and Cyperus involucratus. Ecological Engineering, vol. 35, $\mathrm{n}^{\circ} 2$, p. $238-247,2009$.

[15] MANJATE, E. S.; ZUMALACARREGUI, J. A. G.; VON SPERLING, M. Estudo do comportamento hidraúlico e de balanço de massa dos sólidos em wetlands construídos de escoamento vertical tratando esgotos domésticos. $2^{\circ}$ Simpósio Brasileiro sobre Wetlands Construídos, Curitiba, 2015.

[16] MEULEMAN, A. F. M.; LOGTESTIJN, R. V.; RIJS, G. B. J.; VERHOEVEN, J. T. A. Water and mass budgets of a vertical-flow constructed wetland used for waste water treatment. Ecological Engineering. vol. 20, nº 1, p. 31-44, 2003.

[17] DONG, Z.; SUN, T. A potential new process for improving nitrogen removal in constructed wetlands - Promoting coexistence of partial-nitrification and ANAMMOX. Ecological Engineering, vol. 31, p. 69-78, 2007.

[18] PELISSARI, C.; GUIVERNAU, M.; VIÑAS, M.; SOUZA, S. S.; GARCÍA, J.; SEZERINO, P. H.; ÁVILA, C. Unraveling the active microbial populations involved in nitrogen utilization in a vertical subsurface flow constructed wetland treating urban wastewater. Science of the Total Environment, vol. 584-85, p. 642$650,2017 \mathrm{~b}$

[19] SILVEIRA, D. D.; BELLI FILHO, P.; PHILIPPI, L. S.; KIM, B.; MOLLE, P. Influence of partial saturation on total nitrogen removal in a single-stage French constructed wetland treating raw domestic wastewater. Ecological Engineering, vol. 77, p. 257-264, 2015. 